\title{
REVEALING THE UNSEEN UNIVERSE
}

Astronomy is entering an era in which gravitational waves and neutrinos will be used to complement existing techniques and to uncover the hidden features of our Universe. By Mark Zastrow; illustration by Lucy Reading-Ikkanda

\section{GRAVITATIONAL WAVES}

When two black holes or neutron stars in a binary system spiral towards each other, their massive size causes ripples in space-time known as gravitational waves. The strength of these waves increases as the black holes revolve faster, spiralling towards each other until they merge and there is a fall off in the signal (ringdown). The Universe seems to be awash with these cataclysmic collisions, which astronomers expect to tell them how many black holes and neutron stars there are.
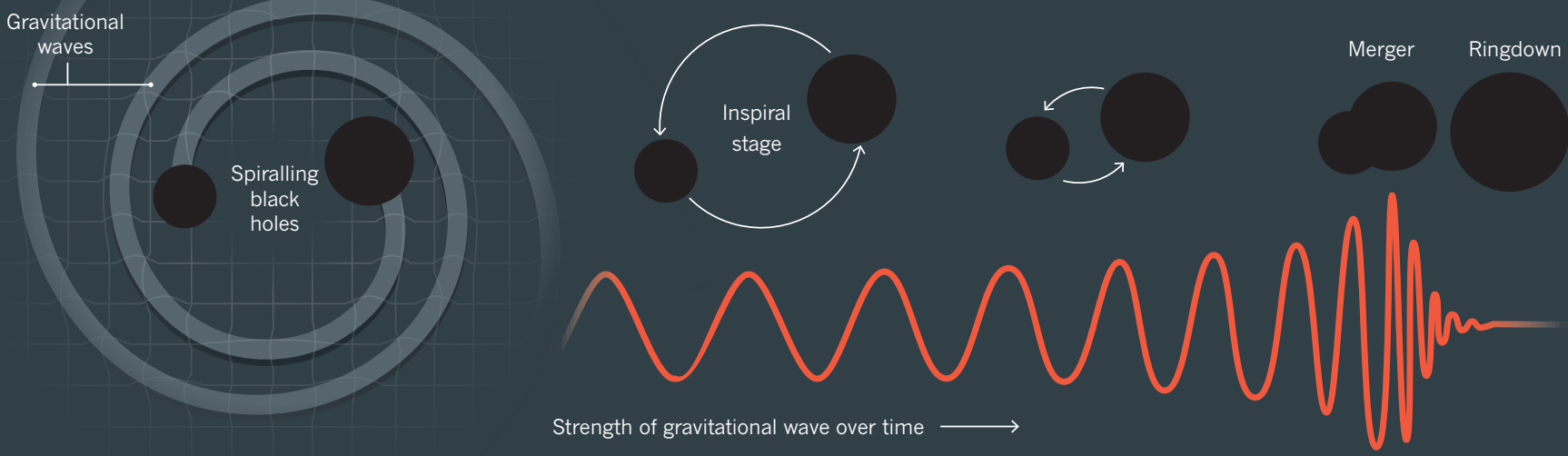

How to detect gravitational waves

In the Laser Interferometer Gravitational-Wave Observatory (LIGO), which detected gravitational waves for the first time in 2015, a laser beam is split in two, and each sent down a 4-kilometre tunnel. The beams are reflected back and forth by mirrors at the end of each tunnel, before being recombined at a detector ${ }^{1}$.

\section{(A)}

\section{Arms} Mirror

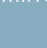

B)

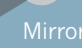

Normal operations

Laser beams travel identical paths and cancel each other out when they recombine at the detector.

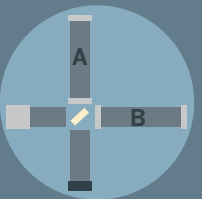

A

B)

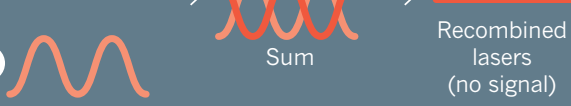

Effect of gravitational waves

The waves warp the region of space-time that the tunnels sit in so that the beams seem to have travelled different distances when they merge. The difference is very small - about the width of an atomic nucleus for the first detection.

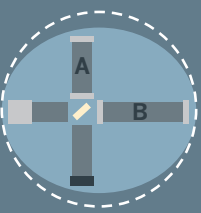

A

B

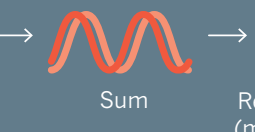

Recombined lasers (measurable signal)

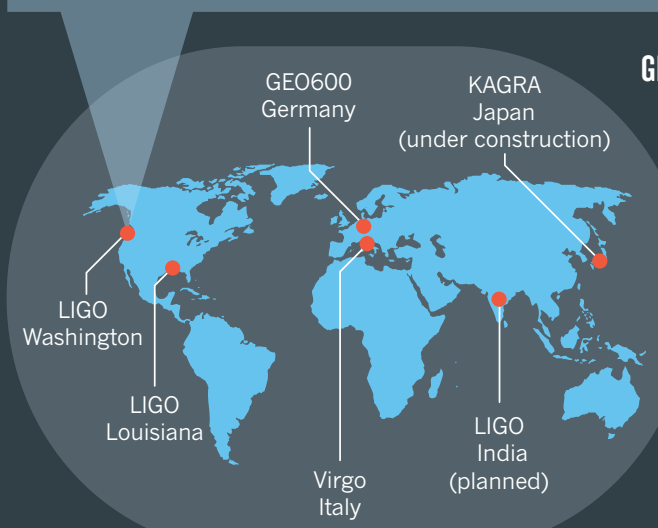

Global network of detectors

There are three operational gravitational wave detectors around the world: two LIGO arrays and Germany's smaller GEO600 facility. Kamioka Gravitational Wave Detector (KAGRA) and Virgo are due to come online in 2018 and 2016, respectively, and a third LIGO detector in India is planned. Combining data from multiple detectors will allow scientists to locate the origin of the waves much more precisely. The laser arms of proposed space-based observatories, such as Europe's eLISA and China's Taiji and TianQin, would be much longer. They could

detect gravitational waves at lower

frequencies and reveal events from weaker sources than can be felt on Earth ${ }^{2}$

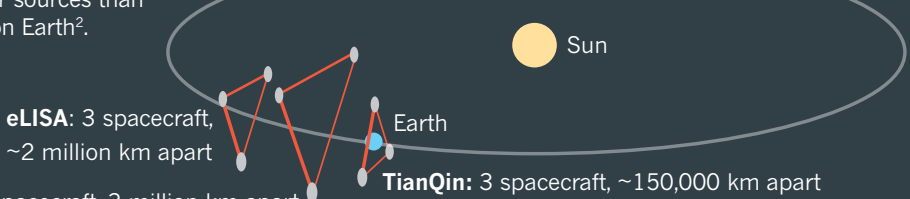




\section{HIGH-ENERGY NEUTRINOS}

Particles known as neutrinos flood the Universe and are so small that they can zip straight through most matter, making them the ideal cosmic messenger. By studying neutrinos, scientists hope to piece together details of the events that made the particles.

Neutrinos produced by the Sun or in nuclear reactors on Earth

have energies of

\section{$1,000,000$ ELECTRONVOLTS}

Neutrinos from outside the Solar System can be a billion

times more energetic, at

\section{$1,000,000,000,000,000$ ELECTRONVOLTS}

The sources of high-energy neutrinos are

shrouded in mystery but the jets of matter streaming from supermassive black holes at the core of active galaxies are a prime suspect.

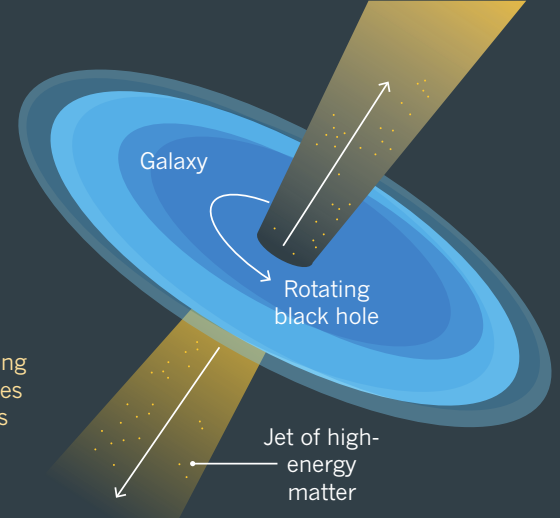

\section{IceCube neutrino observatory \\ Located beneath the \\ Amundsen-Scott South Pole \\ Station, a US research facility, the detector of the IceCube neutrino} observatory is arranged over one cubic kilometre of ice. IceCube's sensitivity is partly due to the fact that ice in the region is one of the purest and most transparent solids on Earth ${ }^{3}$.

The IceTop air-shower detectors filter out some particle 'noise' from cosmic-ray interactions in the atmosphere.

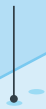

The IceCube laboratory collects and analyses

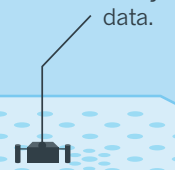

Around 80 steel cables in the ice hold 5,160 digital optical modules (DOMs),

which are sensitive to light

and allow researchers to track the paths of neutrinos.

\section{How leeCube works}

(1) On rare occasions, a neutrino will collide with another particle, such as a proton or neutron, in IceCube's ice.

(2) The collision creates a shower of particles that radiate blue light. Most fizzle out after a few metres, creating a bloom of light. But one particle $-a$ muon - continues through the ice on the neutrino's original path.

3 Optical sensors send data about light intensity to the surface. The pattern (indicated by the colour, size and distribution) of the activated modules allows scientists to determine the neutrino's energy and rough direction.

(4) IceCube can also spot the trails of light left as the muons, produced by neutrino collisions outside the detector, pass through it. But these tracks are also produced by muons from other sources, including cosmic rays. To combat this, IceCube uses Earth as a noise filter, looking only for muons that have passed through the planet.

face
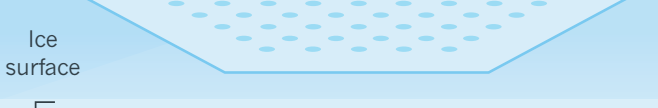

$$
\text { ( }
$$

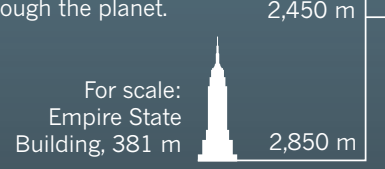

Timeline of high-energy neutrino discovery

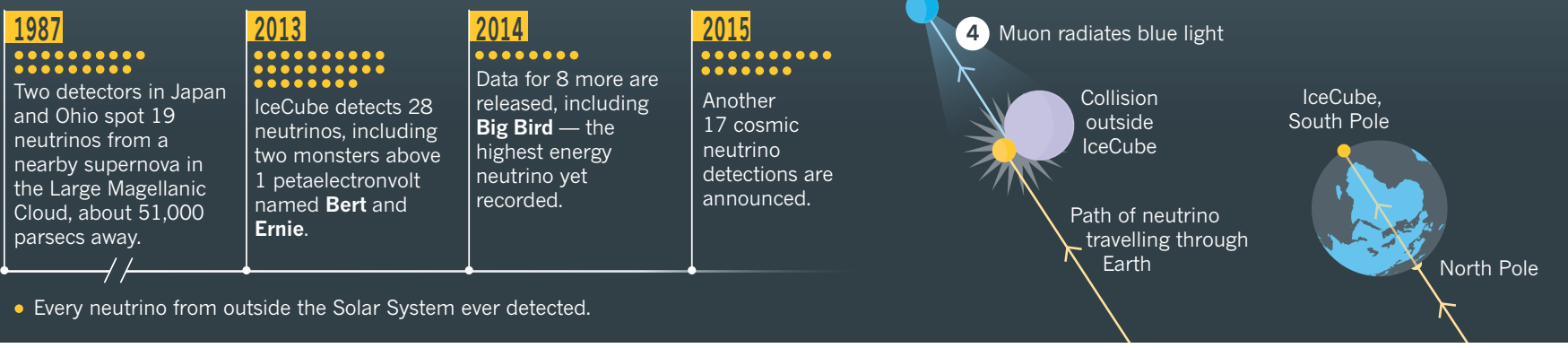

